\title{
Analysis on Intensity Matching Coefficient of Power Distribution Circuit Based on System Reliability Theory
}

\author{
Jiceng Han ${ }^{1}$, Yan Gao ${ }^{2}$, Jianbin Cai ${ }^{1}$, Deyuan Lin ${ }^{1}$, Hongjie Zhang ${ }^{2}$, Yangsen $\mathrm{Li}^{3}$ \\ ${ }^{1}$ Electric Power Research Institute of Fujian Electric Power Company, Fuzhou 350000 \\ ${ }^{2}$ China Electric Power Research Institute, Beijing 100055 \\ ${ }^{3}$ State Grid Fujian Electric Power Company, Fuzhou 350000
}

\begin{abstract}
The design intensities of four parts, i.e. ground conductor, hardware fittings, pole tower and foundation are greatly different, so it is difficult to evaluate the reliability of the whole power distribution circuit exactly. In this thesis, the intensity distribution functions of ground conductor, hardware fittings, pole tower and foundation have been calculated respectively, and then based on the system reliability theory, the reasonable failure sequence of power distribution circuit conforming to economic optimization principle is recommended. Under the assumed failure sequence, the calculation about intensity matching coefficient at the target confidence level is implemented, and finally, the intensity matching coefficients of ground conductor, hardwire fittings, pole tower and foundation of power distribution circuit are given.
\end{abstract}

\section{Introduction}

The power distribution circuit is a complicated engineering system with serial connection of multi-base towers (poles). From the perspective of function, it can be found that the damage or failure of any base tower (pole) will impact the normal running of the circuit system, so the power distribution wire can be regarded as the series system consisting of various base towers. As a sub-section of the whole circuit, the strain section decides the reliability of normal power supply of a greater whole net rack, while the system reliability of power distribution circuit within a strain section is decided by the reliability of series system consisting of four parts, i.e. ground conductor, hardware fittings, pole tower and foundation. With constantly deepened research on reliability of power grid system, the reliability of system consisting of various kinds of elements such as ground conductor, hardwire fittings, pole tower and foundation within a strain section attracts more and more attention.

Through calculating the reliability indexes of insulator, hardware fittings, ground conductor and pole tower in the strain section, Wang Songtao et al.[1] introduced the Stevenson-Moses algorithm for calculating reliability index of series system, and analyzed the reliability index of single tower system. Next, they calculated the reliability indexes of the whole strain section system from 3 situations, i.e. mutually independent among units, completely related among units and incompletely related among units. Zhang Zhuoqun[2] proposed the recursive calculation method for four-level subsystem about calculation of reliability of the power transmission circuit system which crosses G-series high-speed train, and analyzed the reliabilities of a strain section of power transmission circuit of a typical angle steel pole tower and a strain section of power transmission circuit of a typical steel pipe pole tower. His research shows that the recursive calculation method for four-level subsystem is feasible. The system reliability of a strain section of power transmission circuit can be enhanced through enhancing important coefficient of pole tower member design, discounting span of straight line tower or discounting the angle of angle tower. With the reliability, sensitivity and correlation of power transmission tower structure as key research points, Li Huijun[3] researched the sensitive degrees of elastic modulus of member, leg length, leg thickness and other random variables to the maximum deformation and the strain energy under different horizontal load functions, defined the obvious random variables which influence both of them the most, and clearly sorted the correlation between the maximum deformation and the total strain energy. His research shows that when the load level is relatively low, except load, both of them are the most sensitive to the elastic modulus, leg length and leg thickness of vertical pole member at the bottom in the middle; when the load level is relatively high and the plastic development of power transmission tower is relatively sufficient, the maximum deformation of power transmission tower can be reduced effectively through increasing the mean value of yield strength of vertical pole member at the high part in the middle and decreasing its standard deviation, so as to decrease the probability of its collapse damage. According to the Hooke's law, Farzaneh M[4] gave the calculation expression of comprehensive breaking force of conductor, and through experiment, he gave the distribution functions of steel strand, aluminum strand tensile strength and cross 
area; in addition, he simulated the distribution function of comprehensive breaking force of conductor by adopting the Monte Carlo method. Based on the load reliability, Zhang Ganzhou et al.[5] proposed a kind of design method of concrete electric pole, and through the cantilever test, the anti-bending intensity distribution function of electric pole was determined. Ling Sihai[6] believed that the antibending intensity of ring-type concrete electric pole followed the normal distribution, with relatively large dispersity, and after combining the probability density function of anti-bending intensity of electric pole and the extreme value distribution function of wind speed in Hainan Region, he calculated the pole collapse probability of $10 \mathrm{kV}$ power distribution circuit in Hainan Province. In the foreign circuit planning, the IEC60826 is adopted as the criterion of circuit design[7] of circuit design usually. The traditional design method assumes that the load and structural intensity acting on the circuit structure are fixed values, while the IEC 60826 standard introduced the concept of probability design method, emphasizing the uncertainty of structural intensity and the uncertainty of the maximum load which occurs each year, with the structural fault probability as design basis. The IEC 60826 standard rules that after the load reoccurrence period $T$ is determined, the T-year load is recorded as $Q_{T}$. Then, the structure is designed as per the standard that the probability of the structural intensity being less than $Q_{T}$ is $10 \%$. Fenton $\mathrm{G} \mathrm{A}[8]$ analyzed the intensity distribution function of pole tower by adopting the ANSYS software, and based on the IEC standard, he conducted span optimization to the circuit which adopted such pole power, and pointed out that this kind of optimization method could be applied into the planning of other power transmission and distribution circuit.

Based on the research achievements of predecessors, by adopting system reliability theory and according to engineering design experience, this thesis firstly recommended the reasonable failure sequence of power distribution circuit which conforms to economic optimization principle. Under such failure sequence, this thesis implemented the calculation of intensity matching coefficient under specific confidence level, and finally gave relatively reasonable intensity matching coefficients of ground conductor, hardware fittings, pole tower and foundation of power distribution circuit.

\section{Calculation of Intensity of Each Module of Power Distribution Circuit}

\subsection{Calculation of Pole Tower Intensity}

The design of pole tower structure adopts the design method of ultimate limit state with the probability theory as base, and the intensity and stability of structure or member, and the connection intensity, shall undergo load combination according to basic combinations of load effect according to requirements for ultimate limit state of bearing force, and the design is conducted as per the formula (1):

$$
R>\gamma_{0}\left(\gamma_{G} C_{G} G_{\kappa}+\psi \sum \gamma_{Q i} C_{Q i} Q_{i \kappa}\right) / \phi_{s}
$$

In the formula: $R$ - design value of structural member resistance; $\phi_{\mathrm{S}}$ - intensity matching coefficient, taking value as per Table 3.5-1, and if the pole tower is regarded as the module with the minimum reliability, the taken value can be $1.0 ; \gamma_{0}$ - structural importance coefficient; $\gamma_{\mathrm{G}}$ - permanent load partial coefficient, taking the value of 1.0 in case of being in favor of structural; otherwise, taking the value of $1.2 ; \gamma_{\mathrm{Qi}}$ - the partial coefficient of the variable load of the ith item, taking the value of $1.4 ; C_{\mathrm{G}}$ standard value of permanent load; $Q_{\mathrm{iK}}$ - standard value of variable load of the ith item; $\psi$ - combination coefficient of variable load, taking the value of 1.0 in case of normal running condition; taking the value of 0.9 in case of wire breaking condition and installation condition; taking the value of 0.75 in case of checking calculation condition; $G_{\mathrm{K}}$ - standard value of permanent load.

\subsection{Calculation of Intensities of Conductor and Ground Wire}

After the ultimate limit design load acts on the conductor and ground wire, the maximum tension at the lowest point can be worked out. Usually, the maximum tension at the lowest point of sag shall meet requirements in the formula (2):

$$
\frac{T_{\mathrm{P}}}{K_{\mathrm{c}}}>\frac{T_{\max }}{\phi_{S}}
$$

In the formula: $T_{\max }-$ maximum tension $(\mathrm{N})$ at the lowest point of sag of conductor and ground wire; $\phi_{S}$ design of conductor and ground wire by exerting the maximum critical synthetic load on the typical span unit length. At this time, $\phi_{S}=1.0 . T_{\mathrm{P}}$ - breaking force $(\mathrm{N})$ of conductor and ground wire; $K_{\mathrm{c}}$ - design safety coefficient of conductor and ground wire. The design safety coefficient at the lowest point of sag of conductor and ground wire shall not be less than 2.5, and the design safety coefficient of the hanging point shall not be less than 2.25. The design safety coefficient of ground wire shall not be less than the design safety coefficient of conductor.

Under the meteorological conditions at the rare wind speed, $T_{\max }$ shall not exceed $70 \%$ of the breaking force of conductor and ground wire, and the maximum tension of the hanging point shall not exceed $77 \%$ of the breaking force of conductor and ground wire.

\subsection{Calculation of Intensities of Base and Foundation}

The pressure stress of foundation bottom shall meet requirements for the following formula:

$$
\mathrm{f}>\frac{P}{\phi_{S}}
$$


In the formula: $P$ - average pressure standard value $\left(\mathrm{N} / \mathrm{m}^{2}\right)$ acting on the foundation bottom; $f$ - design value of base bearing force; $\phi_{s}$ - intensity matching coefficient, taking the value as per Table 3.5-1.

In case of eccentric loading, besides meeting requirements in the formula (3), it is still required to meet requirements of the formula (4):

$$
1.2 \mathrm{f}>\frac{P_{\max }}{\phi_{S}}
$$

In the formula: $P_{\max }$ - the maximum pressure standard value $\left(\mathrm{N} / \mathrm{m}^{2}\right)$ acting at the edge of foundation bottom;

The calculation of foundation intensity shall be subject to relevant rules specified in DL/T 5219, and the right item of the formula shall be divided by $\phi_{s}$.

Note: In the GB 50061 and DL/T 5219, the coefficient $\gamma_{\mathrm{f}}$ occurs, which shall be taken into consideration when the load is calculated by adopting the partial coefficient method of ultimate limit state design; and it is not required to be taken into consideration in case of adopting allowable stress method.

\subsection{Calculation of Intensities of Insulator and Hardware Fittings}

The intensities of insulator and hardware fittings shall be calculated as per the formula (5):

$$
\frac{F_{u}}{K}>\frac{F}{\phi_{S}}
$$

In the formula: $K$ - mechanical intensity safety coefficient, subject to DL/T 5220; $F$ - design load, KN; $F_{\mathrm{u}}$ - electromechanical damage load of suspension insulator, bending damage load of pin insulator and porcelain cross arm insulator or damage load of shackle insulator and hardware fittings, KN. $\phi_{S}$ - For the variable coefficient COV is less than $7 \%$ usually, for all insulator strings, $\phi_{s}$ $=\phi_{s 2}=0.90$.

\section{Economic Comparison and Selection of Reasonable Failure Sequence}

In this Section, based on the data obtained from above mentioned actual measurement system, the research on average wind speed and turbulence intensity characteristics to the high-altitude regions (open terrain) in Tibet is conducted, the distribution characteristics of average wind speed and turbulence intensity profile and corresponding parameter value taking characteristics are analyzed, and they have been compared with relevant rules in the existing Chinese code in a quantitative way.

\subsection{Characteristics of Average Wind Speed}

After comprehensively considering the construction cost and repair difficulty, and combining the engineering experience, there are three possible failure sequences:

(1) Straight Line Tower, Ground Conductor, Insulator, Hardware Fittings, and Foundation;

For the first kind of failure sequence, the straight line pole collapses, while the ground conductor, insulator, hardware fittings and foundation are intact; the electric pole may be inclined or broken (the root is broken, the middle part is broken and the top is broken), and the ground conductor and insulator also may be intact, but this is the severest situation after failure of electric pole. Generally, the electric pole fails, while other originals are intact, and this kind of damage mode is relatively common in case of natural disaster or man-caused accident.

(2) Ground Conductor, Straight Line Tower, Insulator, Hardware Fittings and Foundation;

The second of situation mainly occurs when there is severe ice coating, at this time, the ground conductor is broken, the straight line tower is intact, the hardware fittings and insulator are intact, and the foundation is also intact.

(3) Hardware Fittings, Straight-line Tower, Ground Conductor, Insulator, and Foundation;

For the third kind of situation, the hardware fittings are damaged, the insulator is intact, the straight line tower is intact, and the ground conductor falls onto the ground but is not damaged, and the foundation is intact. For this kind of situation, unless there is artificial destruction or the material is of severe ageing, the probability of occurrence is very low, for during the design of hardware fittings, a relatively high safety coefficient has been taken into consideration. If the design safety of hardware fittings is lower than that of any other element, this will be very adverse to the circuit structure (for all connections shall be designed as not lower than the intensity of the connection member). Therefore, this situation is not within the comparison scope. The summary of actual situation is as shown in Table 1.

Table 1. Table of Element Damage Situations of Possible

\begin{tabular}{|c|c|c|}
\hline $\mathrm{S} / \mathrm{N}$ & Failure Sequence & Damage Situation \\
\hline 1 & $\begin{array}{l}\text { Straight Line Rod, } \\
\text { Ground Conductor, } \\
\text { Insulator, Hardware } \\
\text { Fittings, and Foundation }\end{array}$ & $\begin{array}{l}\text { Straight Line Rod, } \\
\text { Ground Conductor, } \\
\text { Insulator, Hardware } \\
\text { Fittings, and Foundation }\end{array}$ \\
\hline 2 & $\begin{array}{l}\text { Ground Conductor, } \\
\text { Straight Line Rod, } \\
\text { Insulator, Hardware } \\
\text { Fittings, and Foundation }\end{array}$ & $\begin{array}{l}\text { Ground Conductor, } \\
\text { Straight Line Rod, } \\
\text { Hardware Fittings, and } \\
\text { Foundation }\end{array}$ \\
\hline
\end{tabular}
Failure Sequence

Note: The elements with box in the Table above have been damaged.

For above failure sequence, it is necessary to consider the damage of a strain section, so as to facilitate calculation and analysis. Take the length of strain section as $1 \mathrm{~km}$. The economic calculation includes the circuit loss, repair cost and duration for repair.

(1) Circuit loss. Only the direct loss of circuit is taken into consideration, i.e. the sum of costs of lost rod or lost 
wire. The indirect loss (such as management cost and the generated social influence) is not within the scope of consideration for it cannot be measured exactly.

(2) Repair cost. Only the direct cost generated due to repair to original status is taken into consideration, and the reconstruction cost is not to be taken into consideration.

Table 2. Loss of A Strain Section (1km) of 10kV Typical Scheme (Z-G120CP-NX)

\begin{tabular}{ccccc}
\hline \multirow{2}{*}{ Element } & \multicolumn{2}{c}{ The First Kind of Failure Sequence } & \multicolumn{2}{c}{$\begin{array}{c}\text { The Second Kind of Failure } \\
\text { Sequence }\end{array}$} \\
\cline { 2 - 5 } & Quantity & Loss (RMB) & Quantity & Loss (RMB) \\
\hline Conductor & 0 & 0 & 1.4 & 26600 \\
\hline Concrete Rod & 15.4 & 26180 & 0 \\
\hline Insulator & 0 & 0 & 0 & 0 \\
\hline Hardware Fittings & 0 & 0 & 0 & 0 \\
\hline Galvanized Iron Fittings & 0 & 0 & - & 26600 \\
\hline Total & - & 26180 & 0
\end{tabular}

Table 3. Repair Cost of A Strain Section (1km) of 10kV Typical Scheme

\begin{tabular}{ccc}
\hline \multirow{2}{*}{ Item } & $\begin{array}{c}\text { The First Kind of Failure } \\
\text { Sequence }\end{array}$ & $\begin{array}{c}\text { The Second Kind of Failure } \\
\text { Sequence }\end{array}$ \\
\cline { 2 - 3 } & Repair (RMB) & Repair (RMB) \\
\hline Pole Tower Work & 32514.6 & 0 \\
\hline Stringing Work & 0 & 30257.2 \\
\hline Fittings Work & 2617 & 2617 \\
\hline Total & 35131.6 & 32874.2 \\
\hline
\end{tabular}

Table 4. Duration for Repair of A Strain Section (1km) of 10kV Typical Scheme (Z-G120CP-NX)

\begin{tabular}{ccc}
\hline \multirow{2}{*}{ Item } & The First Kind of Failure Sequence & $\begin{array}{c}\text { The Second Kind of Failure } \\
\text { Sequence }\end{array}$ \\
\cline { 2 - 3 } & Duration for Repair (Work-day) & Duration for Repair (Work-day) \\
\hline Assembled Electric Pole & 2.156 & 0 \\
\hline Vertical Electric Pole & 6.93 & 0 \\
\hline Stringing & 0 & 14.97 \\
\hline Installation of Fittings & 2.156 & 2.156 \\
\hline Total & 11.242 & 17.126 \\
\hline
\end{tabular}

From Table 2 to Table 4, it can be known that: (1) The loss of the first kind of failure sequence (pole collapse) is slightly less than that of the second kind of failure sequence (wire breaking); the repair cost of the first kind of failure sequence (pole collapse) is slightly higher than that of the second kind of failure sequence (wire breaking); therefore, in a word, the sum of costs of the first scheme is close to that of the second scheme. (2) From the perspective of duration for repair, the duration required by the second kind of failure sequence (wire breaking) is obviously greater than that of the first kind of failure sequence (pole collapse).

In case of occurrence of disaster or accident, the most important thing is to recover the circuit to normal running. After comprehensively considering its social benefits, the first kind of failure sequence, which requires the shortest repair duration is recommended, i.e. the reliability of electric pole is lower than that of any other element.

\section{Calculation of Intensity Matching Coefficient of Each Component of Power Distribution Circuit}

Establish a probability model that the target probability of module 2 exceeds the target probability of module 1 , for example, the probability of $R_{2}>R_{1}$ is $95 \%$ or other guaranteed probability:

$$
P\left(R_{2}-R_{1}>0\right)=0.95=P(\text { sof })
$$

In the formula: $R_{2}$ and $R_{1}$ are module intensities, and $P$ (sof) refers to the intensity matching and failure sequence probabilities. 
After adopting statistical method, for different combinations of $v_{R 1}$ and $v_{R 2}\left(v_{R 1}\right.$ and $v_{R 2}$ are intensity variable coefficients), the intensity matching coefficient $\phi_{s}$ can be inferred. $\phi_{s}$ is related to the exceedance probability of intensity of the module which is adopted for comparison:

$$
\phi_{s}=\frac{(10 \%) R_{1}}{(10 \%) R_{2}}
$$

Assume that the probability density function of intensity follows the normal distribution. Then, the guaranteed probability of obtaining intensity matching target can be expressed with the reliability index $\beta_{\text {sof }}$ :

$$
F\left(\beta_{\text {sof }}\right)=P_{\phi}
$$

From the Table of Normal Distribution Probability, the reliability index $\beta_{\text {sof }}$ which corresponds to the failure probability can be obtained.

According to the reliability principle:

$$
\beta_{\text {sof }}=\frac{\bar{R}_{1}-\bar{R}_{2}}{\sqrt{\sigma_{R_{1}}^{2}+\sigma_{R_{2}}^{2}}}
$$

Standard deviation $\sigma_{R_{1}}=v_{R_{1}} \times \bar{R}_{1} \quad, \quad$ standard deviation $\sigma_{R_{2}}=v_{R_{2}} \times \bar{R}_{2}$; both $\bar{R}_{1}$ and $\bar{R}_{2}$ are

Table 5. Intensity Matching Coefficients in case that Failure of Module $R_{2}$ Later than Module $R_{1}\left(P_{\Phi}=0.95\right)$

\begin{tabular}{lllllllll}
\hline & & \multicolumn{5}{c}{$R_{1}$ Variable Coefficient } \\
\hline & & 0.03 & 0.05 & 0.075 & 0.10 & 0.15 & 0.20 \\
\cline { 2 - 8 }$R_{2}$ Variable Coefficient & 0.03 & 0.91 & 0.88 & 0.81 & 0.75 & 0.63 & 0.53 \\
\cline { 2 - 8 } & 0.05 & 0.92 & 0.89 & 0.83 & 0.76 & 0.65 & 0.54 \\
\cline { 2 - 9 } & 0.10 & 0.94 & 0.90 & 0.85 & 0.79 & 0.68 & 0.57 \\
\cline { 2 - 8 } & 0.15 & 0.94 & 0.91 & 0.86 & 0.81 & 0.70 & 0.60 \\
\cline { 2 - 8 } & 0.20 & 0.94 & 0.91 & 0.86 & 0.81 & 0.71 & 0.61 \\
\cline { 2 - 8 } & 0.30 & 0.95 & 0.91 & 0.87 & 0.82 & 0.72 & 0.63 & 0.64 \\
\hline
\end{tabular}

Table 6. Value Taking of Intensity Variable Coefficient

\begin{tabular}{cc}
\hline Module & Intensity Variable Coefficient (COV) \\
Conductor and Ground Wire & 0.03 \\
Hardware Fittings & 0.05 \\
Insulator & 0.05 \\
Steel Pipe Rod & 0.05 \\
Concrete Rod & 0.15 \\
Wooden Rod & 0.20 \\
Lattice Iron Tower & 0.10 \\
Rock Anchor Rod Foundation & 0.10 \\
Pile Foundation & 0.25 \\
Foundation with Excavation or Mechanical Compaction Back & 0.20 \\
Filling Soil & 0.30 \\
Foundation with Non-compacted Backfilling Soil &
\end{tabular}

During the overall circuit design, according to variable coefficients among different modules, the intensity average values of module intensities. Introducing the center safety coefficient:

$$
\alpha=\frac{\bar{R}_{2}}{\bar{R}_{1}}
$$

The formula (8) is changed into:

$$
\beta_{\text {sof }}=\frac{1-\alpha}{\sqrt{\alpha^{2} \times v_{R_{1}}^{2}+v_{R_{2}}^{2}}}
$$

From the formula (10), it can be known that the center safety coefficient $\alpha$ can be worked out through solving the following quadratic equation:

$$
\alpha^{2}\left[1-\left(\beta_{\text {sof }} v_{R_{1}}\right)^{2}\right\rfloor-2 \alpha+\left\lfloor 1-\left(\beta_{\text {sof }} v_{R_{2}}\right)^{2}\right\rfloor=0
$$

Table 5 gives the calculation results when $\beta_{\text {sof }}=1.645$ while the variable coefficients $v_{R_{1}}$ and $v_{R_{2}}$ take different values. See Table 6 for the values of variable coefficients.

$$
\phi_{s}=\frac{(5 \%) R_{1}}{(5 \%) R_{2}}=\frac{\bar{R}_{1}\left(1-1.645 v_{R_{1}}\right)}{\bar{R}_{2}\left(1-1.645 v_{R_{2}}\right)}=\frac{1 \times\left(1-1.645 v_{R_{1}}\right)}{\alpha \times\left(1-1.645 v_{R_{2}}\right)}
$$




\section{Conclusions}

Based on the comprehensive comparison and analysis on economy and social effect, this thesis firstly determined the optimal element failure sequence of power distribution circuit under the actual using situation, and then determined the intensity matching coefficients corresponding to different variable coefficients of various modules based on the method for analysis on system reliability probability, so it is meaningful in guiding the enhancement of economy and safety of design scheme of power distribution circuit.

\section{Acknowledgments}

This work has been funded by the technology project of State Grid Fujian Electric Power Company (Project number: 52130417000T). The authors would like to thank the sponsor of State Grid Fujian Electric Power Company.

\section{References}

1. Wang Songtao, Wu Haiyang, Yang Jingsheng, Bao Yongzhong. Analysis on Reliability of Strain Section Tower Line System of Power Transmission Circuit[J]. China Electric Power (Technology Edition), 2014(10):65-69.

2. Zhang Zhuoqun, Li Hongnan, Gong Jinxin, Zhang Ziyin, Li Jiaxiang. Reliability Analysis of Tension Section in Transmission Line $[\mathrm{J}]$. Electric Power Construction, 2014, 35(05):42-49.

3. Li Huijun, Xu Shibo, Tang Ye, Wang Wei, Li Qiangqiang. Reliability and Sensitivity Evaluation of Transmission Power Tower[J]. Steel Construction, 2016, 31(09):39-45.

4. Farzaneh M, Savadjiev K. Evaluation of tensile intensity of ACSR conductors based on test data for individual strands[J]. Power Delivery, IEEE Transactions on, 2007, 22(1): 627-633.

5. Zhang Ganzhou. Reliability-based Design of Utility Pole Structure [J]. International Electric Power for China, 2005, 9(4): 51-53.

6. Ling Sihai. Calculation on Reliability of $10 \mathrm{kV}$ Overhead Power Lines $[\mathrm{J}]$. Electric Power Construction, 2010, 22(3): 19-0.

7. International Electrotechnical Commission. IEC 60826 (2003) Design criteria of overhead transmission lines $[\mathrm{J}]$. Geneva, Switzerland-IEC, 2003.

8. Fenton G A, Sutherland N. Reliability-Based Transmission Line Design [J]. Power Delivery, IEEE Transactions on, 2011, 26(2): 596-606. 